\title{
Non-self recognition by monocytes initiates allograft rejection
}

\author{
Martin H. Oberbarnscheidt, ${ }^{1,2}$ Qiang Zeng, ${ }^{1,2}$ Qi Li, ${ }^{1,2}$ Hehua Dai, ${ }^{1,2}$ Amanda L. Williams, ${ }^{1,2}$ Warren D. Shlomchik, ${ }^{3}$ \\ David M. Rothstein, ${ }^{1,2,4,5}$ and Fadi G. Lakkis ${ }^{1,2,4,5}$ \\ 'Thomas E. Starzl Transplantation Institute, University of Pittsburgh School of Medicine and University of Pittsburgh Medical Center, Pittsburgh, Pennsylvania, USA. ${ }^{2}$ Department of Surgery, \\ University of Pittsburgh School of Medicine, Pittsburgh, Pennsylvania, USA. ${ }^{3}$ Departments of Medicine and Immunobiology, Yale University School of Medicine, New Haven, Connecticut, USA. \\ ${ }^{4}$ Department of Immunology and ${ }^{5}$ Department of Medicine, University of Pittsburgh School of Medicine, Pittsburgh, Pennsylvania, USA.
}

\begin{abstract}
Maturation of T cell-activating APCs directly links innate and adaptive immunity and is typically triggered by microbial infection. Transplantation of allografts, which are sterile, generates strong $T$ cell responses; however, it is unclear how grafts induce APC maturation in the absence of microbial-derived signals. A widely accepted hypothesis is that dying cells in the graft release "danger" molecules that induce APC maturation and initiate the adaptive alloimmune response. Here, we demonstrated that danger signals associated with dying cells are not sufficient to initiate alloimmunity, but that recognition of allogeneic non-self by the innate immune system is required. In WT as well as in T cell-, B cell-, and innate lymphoid cell-deficient mice, allogeneic grafts elicited persistent differentiation of monocytes into mature DCs that expressed IL-12 and stimulated T cell proliferation and IFN- $\gamma$ production. In contrast, syngeneic grafts in the same mice elicited transient and less pronounced differentiation of monocytes into DCs, which neither expressed IL-12 nor stimulated IFN- $\gamma$ production. In a model in which $\mathrm{T}$ cell recognition is restricted to a single foreign antigen on the graft, rejection occurred only if the allogeneic non-self signal was also sensed by the host's innate immune system. These findings underscore the importance of innate recognition of allogeneic non-self by monocytes in initiating graft rejection.
\end{abstract}

\section{Introduction}

The mammalian innate immune system recognizes non-self molecules unique to microbial organisms $(1,2)$. This recognition step triggers a rapid inflammatory response and, of paramount importance to the survival of the host, induces the differentiation of myeloid cells into mature APCs. APCs in turn activate the adaptive immune system by presenting microbial antigens and by providing costimulation to $\mathrm{T}$ cells, leading to immunity against infection.

In addition to mounting strong antimicrobial responses, all studied mammals generate potent immunity to cells or tissues of other members of the same species (alloimmunity) $(3,4)$. This is best illustrated by organ and bone marrow transplantation in the clinic (5), but is also observed under natural conditions such as the maternal response to the allogeneic fetus and the rejection of transmissible allogeneic tumors (6-9). Unlike microbial infection, however, it is unclear how allografts, which are sterile, cause the maturation of APCs. A widely accepted paradigm, known as the "danger hypothesis," proposes that necrotic cells or danger-associated molecules released by dying cells in the transplanted organ induce the maturation of APCs that then activate the adaptive alloimmune response $(10,11)$. Danger molecules are diverse cell products that cause inflammation by signaling through TLRs or inflammasomes $(11,12)$. Examples include uric

Conflict of interest: The authors have declared that no conflict of interest exists Submitted: January 24, 2014; Accepted: May 15, 2014.

Reference information: J Clin Invest. 2014;124(8):3579-3589. doi:10.1172/JCI74370. acid and the high-mobility group box 1 (HMGB1) nuclear protein, both of which potentiate $\mathrm{T}$ cell responses when present in supraphysiological amounts in the extracellular space (13-15).

Although danger molecules enhance immune responses, it is unclear whether they are necessary or sufficient for triggering alloimmunity. Initial experiments had shown that deletion of the adaptor molecule MyD88, which is required for signaling by most TLRs, prevents the rejection of single minor antigen-mismatched grafts (16), but later studies failed to demonstrate significant retardation of allograft rejection if the donor and recipient differed by major or multiple minor histocompatibility antigens (17-20). Moreover, allografts parked for an extended period of time in $\mathrm{T}$ cell-deficient hosts to allow the resolution of tissue injury that occurs at the time of transplantation were uniformly rejected when the host was replenished with T cells (21-25). These observations raise the possibility that additional triggers of APC maturation and activation of the adaptive alloimmune response exist.

We have previously shown that mouse monocytes mount a greater inflammatory response to allogeneic cells than to syngeneic cells, suggesting that they are capable of distinguishing between self- and non-self tissues (26). It is not known, however, whether such innate sensing causes APC maturation, initiates the $\mathrm{T}$ cell response, and triggers graft rejection. Here, we addressed this question by analyzing the innate response of wild-type and $\mathrm{T}, \mathrm{B}$, and innate lymphoid cell-deficient mice to either syngeneic or allogeneic heart, kidney, and bone marrow grafts. We demonstrate that allogeneic grafts induced persistent differentiation of host monocytes into mature DCs that produce IL-12 and drive 


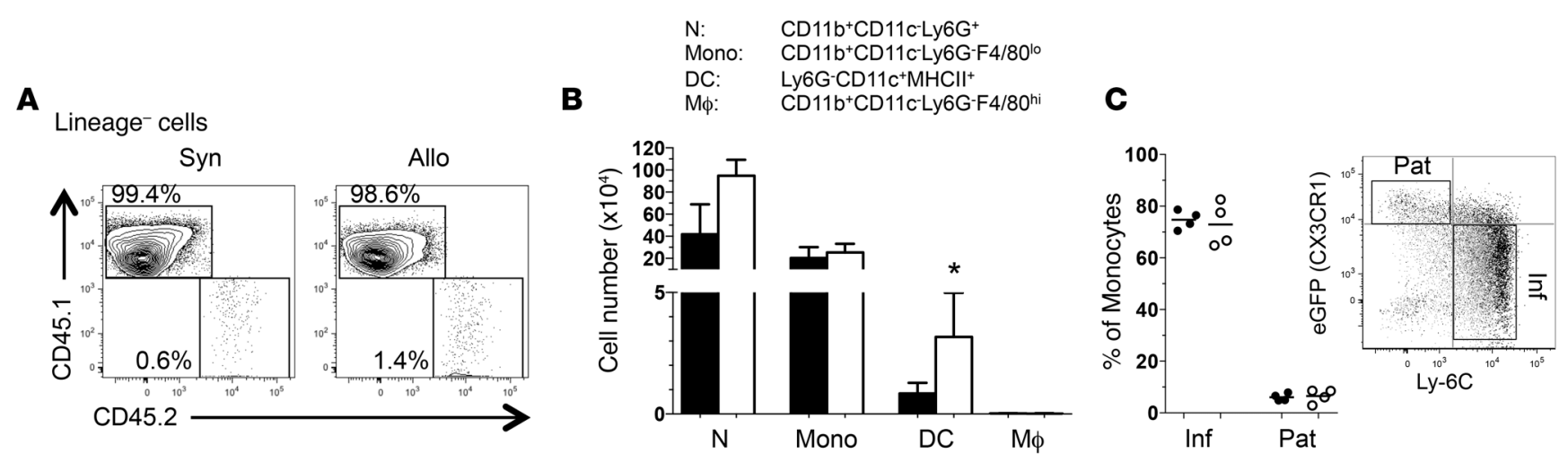

D

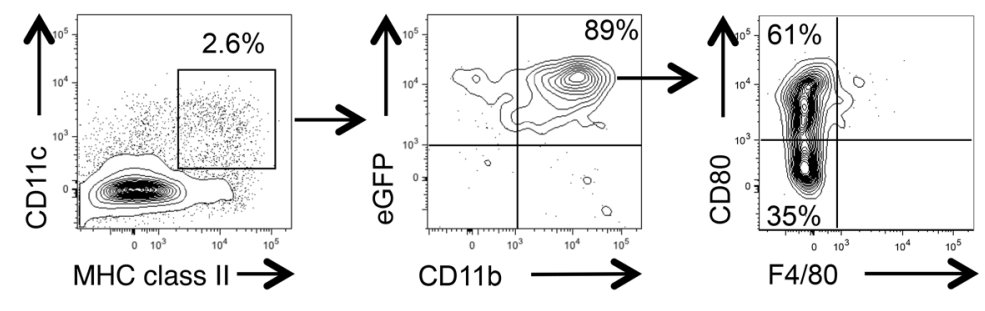

E

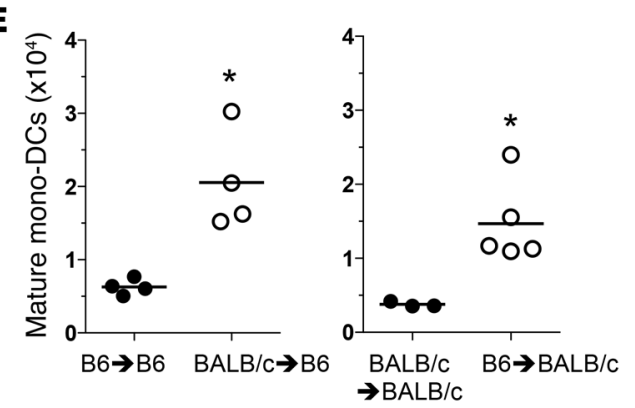

Figure 1. Analysis of myeloid cell infiltrate in heart grafts transplanted into immunocompetent recipients. B6 (syngeneic) or BALB/C (allogeneic) CD45.2 heart grafts were transplanted into $\mathrm{B} 6 \mathrm{CX}_{3} C R \mathrm{1}^{\mathrm{gfp} /+} \mathrm{CD} 45.1$ recipients, except where indicated. Infiltrating cells were analyzed by flow cytometry 1 day after transplantation; $n=4$ grafts/group. (A) Representative flow plot depicting lineage- (CD19-CD90-NK1.1-DX5-) recipient (CD45.1 $1^{+}$and donor (CD45.2+) myeloid cells present in syngeneic (Syn) and allogeneic (Allo) grafts. (B) Enumeration of recipient myeloid populations in syngeneic (black bars) and allogeneic (white bars) grafts. Cell surface markers used to identify cell populations are shown in the text box and in the gating strategy in Supplemental Figure 1. N, neutrophils; Mono, monocytes; DC, dendritic cells; M $\phi$, macrophages. (C) Enumeration and representative flow plot of monocyte subsets (Inf, inflammatory Ly-6C ${ }^{\text {hi } C X 3 C R}{ }^{\text {lo }}$; Pat, patrolling Ly- $6 C^{10} C X 3 C R 1^{\text {hi }}$ ) in syngeneic (black circles represent black events) and allogeneic (white circles represent gray events) grafts. (D) Representative flow plot depicting phenotype of recipient-derived DCs in an allogeneic graft. (E) Enumeration of mature (CD80 $)$ mono-DCs in syngeneic (black circles) and allogeneic (white circles) grafts. Each data point represents 1 graft. Donor-recipient strain combination is indicated on the $x$ axis. ${ }^{*} P<0.05$.

$\mathrm{T}$ cell proliferation and IFN- $\gamma$ production. In contrast, syngeneic grafts elicited transient and less pronounced differentiation of monocytes into DCs that neither expressed IL-12 nor stimulated IFN- $\gamma$ production. In a model in which $\mathrm{T}$ cell recognition is restricted to a single foreign antigen on the graft, rejection occurred only if allogeneic non-self was also sensed by the host's innate immune system. These findings indicate that danger signals alone are not sufficient for inducing an optimal alloimmune response, but that innate recognition of allogeneic non-self is required.

\section{Results}

Heart allografts harbor a greater number of monocyte-derived DCs than do syngeneic grafts. To test whether allogeneic non-self triggers APC maturation in vivo, we first investigated whether monocytes, which are the principal source of APCs in inflamed and infected tissues (27), differentiate into DCs in grafts transplanted into immunocompetent hosts. We transplanted BALB/c (allogeneic) or B6 (syngeneic) CD45.2 hearts into transgenic $\mathrm{B} 6 \mathrm{CX}_{3} C R 1^{g f p /+} \mathrm{CD} 45.1$ mice, in which 1 copy of the fractalkine receptor $\left(C_{3} C R 1\right)$ has been replaced by the $e G f p$ gene (28). Since $\mathrm{CX}_{3} \mathrm{CR} 1$ is ubiquitously expressed on monocyte lineage cells, host monocytes and monocyte-derived cells could be identified by flow cytometry using eGFP and monocyte lineage-specific markers (29). We found that a large number of host (CD45.1 $1^{+}$myeloid cells infiltrated both syngeneic and allogeneic heart grafts within 1 day af- ter transplantation (Figure 1A). The infiltrate consisted of neutrophils, monocytes, DCs, and a small number of macrophages $\left(<1 \times 10^{3}\right.$ cells/graft) (Figure 1B and Supplemental Figure 1; supplemental material available online with this article; doi:10.1172/JCI74370DS1). Analysis of infiltrating monocytes revealed that the majority were Ly6C ${ }^{\text {hi }} \mathrm{CX}_{3} \mathrm{CR}^{\text {lo }}$ (so-called inflammatory monocytes), while the minority were $\mathrm{Ly} 6 \mathrm{C}^{\text {hi }} \mathrm{CX}_{3} \mathrm{CR} 1^{\text {hi }}$ (so-called patrolling monocytes) in both allogeneic and syngeneic grafts (Figure 1C). There was no differential recruitment of one subset or the other to allogeneic grafts (Figure 1C). DC analysis showed that the vast majority were of monocytic origin $\left(\mathrm{eGFP}+\mathrm{CD} 11 \mathrm{~b}^{+}\right)$, lacked the macrophage marker F4/80, and approximately $60 \%$ had acquired the maturation marker CD80 (Figure 1D). Total DCs, total monocyte-derived DCs (mono-DCs), as well as mature mono-DCs were present in significantly larger numbers in allogeneic grafts than in syngeneic grafts (Figure 1, B and E). The same result was observed when the reverse transplantation experiment was performed (B6 hearts transplanted into BALB/c recipients) (Figure $1 \mathrm{E})$, ruling out the potential effect of strain background on monocyteto-DC differentiation. These results provide evidence for exaggerated monocyte differentiation into mature DCs in allogeneic grafts.

Increased numbers of mono-DCs in allografts occur independently of recipient and donor $T, B$, and innate lymphoid cells. To determine whether increased differentiation of monocytes into DCs in allografts is an innate event that occurs independently of the adaptive 

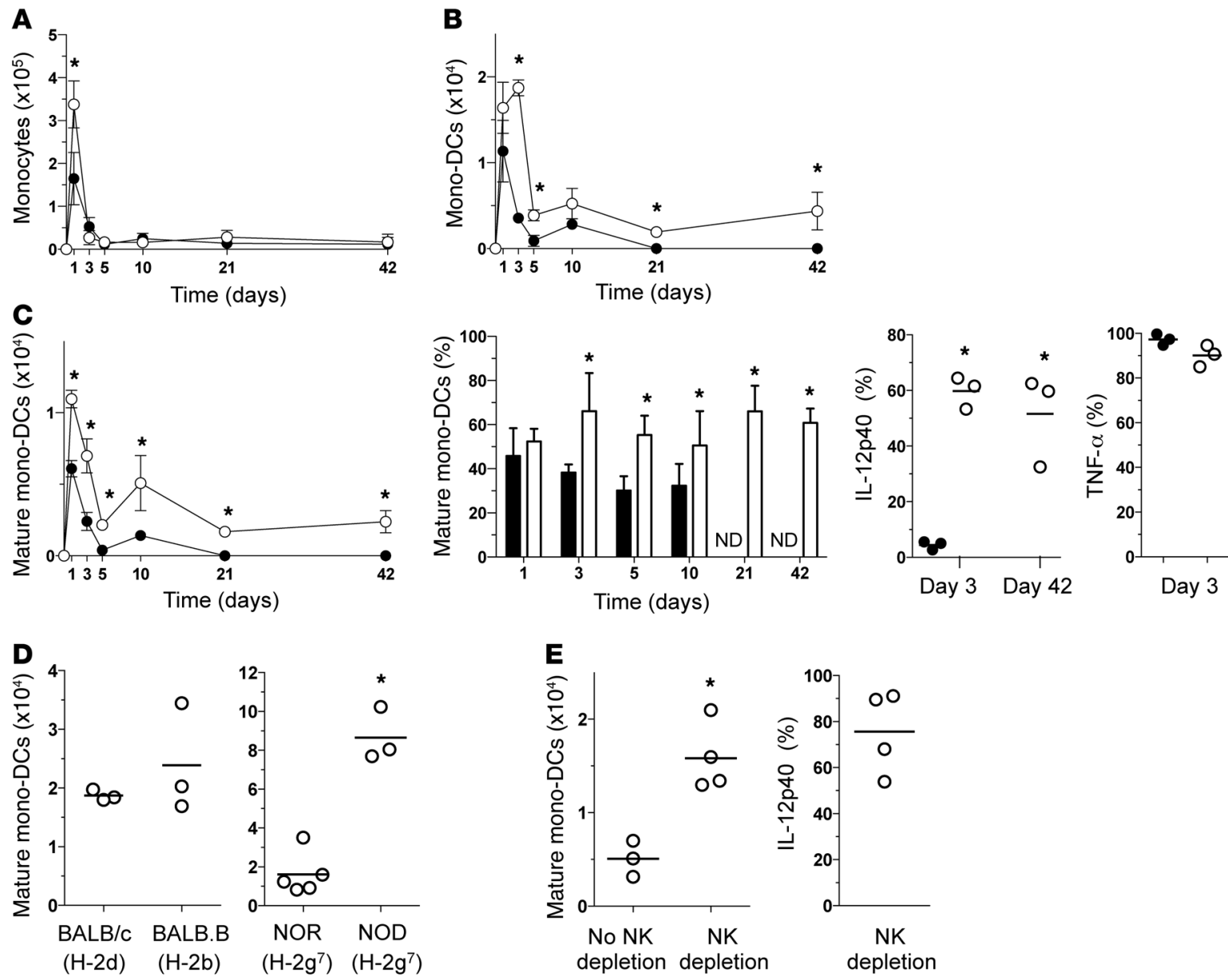

Figure 2. Analysis of monocyte and mono-DC infiltrate in heart grafts transplanted into lymphoid cell-deficient recipients. B6 $R A G^{-1-}$ (syngeneic) or BALB/c $R A G^{-/-}$(allogeneic) heart grafts, except where indicated, were transplanted into B6 $R A G^{-1-} \gamma C^{-1-} C X_{3} C R 1^{9 f / /+}$ recipients. Infiltrating cells were analyzed by flow cytometry at multiple time points after transplantation. (A-C) Enumeration of host monocytes (A), total mono-DCs (B), and mature $\left(C D 80^{+}\right)$mono-DCs (C) in syngeneic (black circles) and allogeneic (white circles) grafts. Percentage of mature mono-DCs and percentage of mature mono-DCs that are IL-12p40+ or TNF- $\alpha^{+}$are shown in C. Data represent the mean \pm SD; $n=4$ grafts/group/time point for days $1,5,10,21$, and $42 . n=6$ grafts/group for day 3. ND, none detected. (D) Effect of donor MHC (BALB/c, H-2d vs. BALB.B, H-2b) and donor non-MHC (NOR, H-2 $g^{7}$ vs. NOD, H-2g ) genotype on the number of mature host mono-DCs in heart allografts 3 days after transplantation into B6 mice (H-2b). (E) Effect of NK depletion in the donor on the number of host-derived mature mono-DCs and their IL-12 production in heart allografts 10 days after transplantation. Same donorrecipient strain combination as in $\mathbf{A}-\mathbf{C} .{ }^{*} P<0.05$.

immune system, we first transplanted BALB/c (allogeneic) or $B 6$ (syngeneic) $R A G^{-/-}$hearts into $B 6 R A G^{-/ \gamma} c^{-/-} C X_{3} C R 1^{\text {sfo/ } /+}$ recipients, which lack T, B, and innate lymphoid cells including NK cells. We analyzed the myeloid cells infiltrating the grafts on days 1,3 , $5,10,21$, and 42 after transplantation. Graft infiltration with host monocytes peaked on day 1 , with 2 -fold more monocytes detected in allogeneic grafts than in syngeneic grafts, but declined to comparable numbers in both groups by day 3 (Figure 2A). Mono-DCs were more abundant in allogeneic grafts than in syngeneic grafts at early time points (days 1-10) and persisted at later time points (days 21 and 42) only in allografts (Figure 2B). Moreover, allografts harbored a significantly greater number and proportion of mature mono-DCs, the majority of which were IL- $12 \mathrm{p} 4 \mathrm{O}^{+}$both early (day 3 ) and late (day 42) after transplantation (Figure 2C). In contrast, mature mono-DCs were less frequent in syngeneic grafts, and only a small fraction $(<5 \%)$ expressed IL-12p 40 (Figure $2 \mathrm{C}$ ). We also tested TNF- $\alpha$ expression by these cells on day 3 and found the ma- jority to be positive, whether isolated from syngeneic grafts or allogeneic grafts (Figure 2C). As in immunocompetent hosts (Figure 1B), no differences in neutrophil, macrophage, or conventional DC (CD11b- DCs) numbers were observed between the 2 graft types (Supplemental Figure 2). In addition, BALB.B grafts, which share MHC loci $\left(\mathrm{H}-2^{\mathrm{b}}\right)$ with $\mathrm{B} 6$ but are otherwise genetically identical to $\mathrm{BALB} / \mathrm{c}$ mice, had numbers of mature mono-DCs similar to those of BALB/c grafts, while NOR and NOD grafts, which share MHC loci $\left(\mathrm{H}-2^{\mathrm{g}^{\mathrm{g}}}\right)$ but are dissimilar at approximately $11 \%$ of the genome outside the MHC locus, elicited strikingly different magnitudes of mature mono-DC infiltrates after transplantation into B6 mice $(\sim 5$-fold greater in NOD grafts than in NORgrafts) (Figure 2D). This indicates that an MHC mismatch between donor and recipient is not necessary for the monocytic response to allogeneic non-self, but rather that genetic mismatches outside the MHC likely determine the response. Therefore, allogeneic tissues elicit persistent differentiation of monocytes into mature, IL-12p $40^{+}$DCs independently 


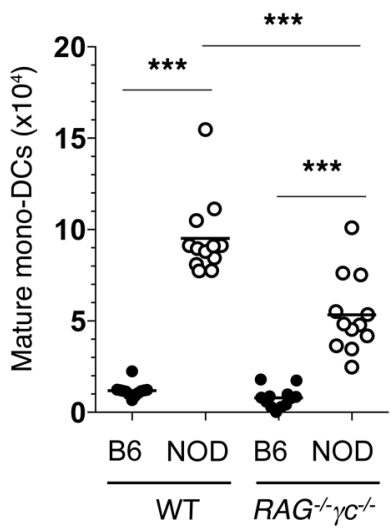

Figure 3. Enumeration of mature mono-DC infiltrate in bone marrow plug grafts transplanted into lymphoid cell-deficient recipients. B6 and NOD grafts on the WT or $R A G^{-/-} \gamma C^{-/-}$background were transplanted under the contralateral kidney capsules of $\mathrm{B} 6 R A G^{-/-} \gamma c^{-/-}$recipients. Mature mono$D C s$ that infiltrated the grafts were enumerated 1 week later. Each data point represents 1 graft; $n=6$ grafts/experiment; each experiment was performed twice. Donor strains are indicated on the $x$ axis. ${ }^{* *} P<0.001$.

of host T, B, and innate lymphoid cells and independently of MHC disparities between donor and host. Our findings also show that syngeneic grafts, in contrast, induce transient monocyte differentiation, and the resulting mono-DCs are IL-12p40-

Transplanted tissues contain donor or passenger lymphoid cells that react to allogeneic non-self on host cells (30). This raises the possibility that enhanced differentiation of host monocytes into DCs elicited by $R A G^{-/}$allografts, which lack T and B cells but have NK cells, may have resulted indirectly from passenger NK cells responding to missing self in the allogeneic host. We therefore transplanted hearts from NK-depleted BALB/c $R A G^{-/-}$donors into $\mathrm{B} 6 \mathrm{RAG}^{-/-} \mathrm{\gamma c}^{-/-} \mathrm{CX} \mathrm{C}_{3} \mathrm{CR} 1^{g f p /+}$ recipients and enumerated mature mono-DCs in the grafts 10 days later. As shown in Figure 2E, NK depletion of the donor did not reduce the number of mature mono-DCs; instead, more were detected than in the nondepleted grafts. IL-12p40 expression by mono-DCs in NK-depleted grafts (Figure 2E) was similar to that observed in the nondepleted grafts shown in Figure 2C. These data indicate that enhanced host monocyte differentiation into DCs after allotransplantation is not an indirect consequence of passenger NK cells reacting to the host. To the contrary, passenger NK cells may downregulate the host monocytic response. To more definitively rule out the possibility that donor NK cells or other innate lymphoid cells trigger the host monocyte response, we used a bone marrow plug transplantation model (whereby the graft is rich in donor lymphoid cells) and compared the host response with that of wild-type versus $R A G^{-/-} \gamma c^{-/-}$grafts. B6 (syngeneic) and NOD (allogeneic) grafts on the wild-type or $R A G^{-/-} \gamma c^{-/-}$background were transplanted under the contralateral kidney capsules of B6 $R A G^{-/-} \gamma c^{-/-}$recipients. Host mature mono-DCs were enumerated in the grafts 1 week later. As shown in Figure 3, significantly greater numbers of mono-DCs were present in wild-type and $R A G^{-/-} \gamma c^{-/-}$allografts than in corresponding syngeneic grafts, indicating that the host monocyte response to allogeneic non-self occurs in the complete absence of both donor and recipient adaptive and innate lymphoid cells. However, we did observe a significant difference in mono-DC numbers between wild-type and $R A G^{-/-} \gamma c^{-/-}$allografts, implying that donor lymphoid cells contribute to the host monocyte response in this model but are not required for eliciting it. These findings therefore establish the presence of a bona fide innate response to allogeneic non-self in the mouse characterized by the differentiation of monocytes into mature DCs.

Mono-DCs are increased in kidney allografts. To investigate whether the monocyte response to heart allografts also occurs in other types of solid organ transplants, we performed 2-photon intravital imaging of syngeneic and allogeneic kidney grafts transplanted into $R A G^{-/-} \gamma \mathrm{C}^{-/-} C X_{3} C R 1^{g f p /+}$ recipients and quantified monocyte entry and differentiation into DCs in the graft tissue. As shown in Figure $4 \mathrm{~A}$, $\mathrm{eGFP}^{+}$cells, which represent recipient monocyte-lineage cells (29), were observed in equal numbers in syngeneic and allogeneic grafts immediately after transplantation (day 0). We detected the vast majority inside the capillaries (Fig-
A

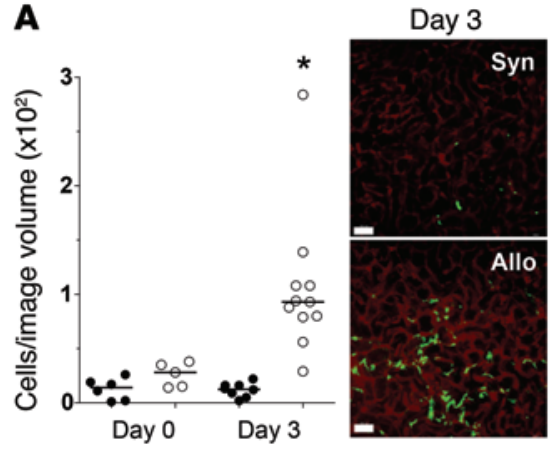

B

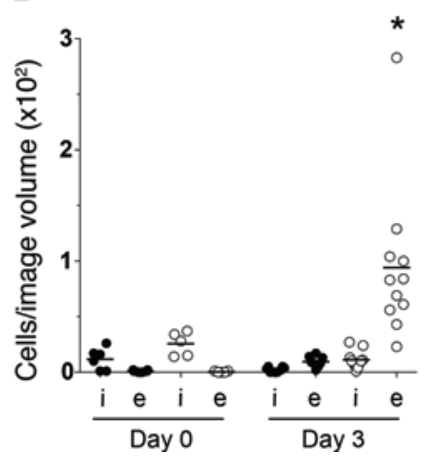

C

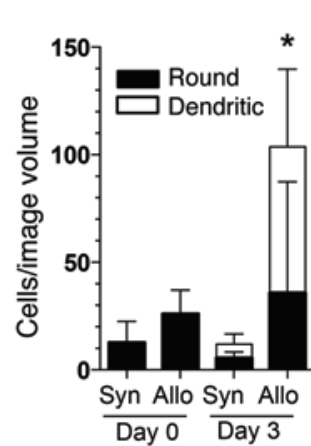

Day 3

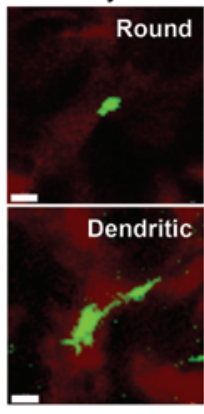

Figure 4. Analysis of monocyte and mono-DC infiltration of kidney grafts transplanted into lymphoid cell-deficient recipients. B6 (Syn; black circles) or $\mathrm{BALB} / \mathrm{c}$ (Allo; white circles) kidneys were transplanted into B6 $R A C^{-1-} \gamma C^{-1-} C X_{3} C R 7^{g f p /+}$ mice. Kidneys were imaged by intravital 2-photon microscopy either immediately (day 0 ) or on day 3 after transplantation. Enumeration of total (A), intravascular (i), and extravascular (e) (B), and round and dendrite-shaped $\mathrm{GFP}^{+}$cells (C) in kidney grafts. Each data point in $\mathbf{A}$ and $\mathbf{B}$ represents 1 image volume. Image volume $=510 \times 510 \times 25 \mu \mathrm{m}$. Representative micrographs show $\mathrm{GFP}^{+}$cells in green and capillaries in red. Scale bars: $50 \mu \mathrm{m}$ (A) and $10 \mu \mathrm{m}$ (C). $n=3-4$ mice/group (1-5 image volumes/mouse). ${ }^{*} P<0.05$ compared with the corresponding syngeneic group. 
A
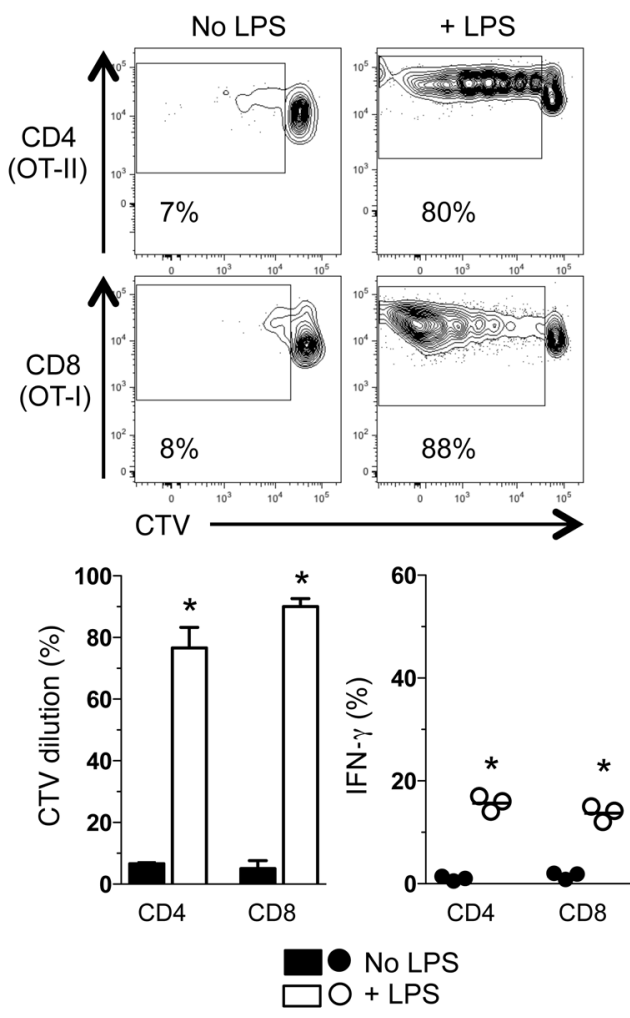

B
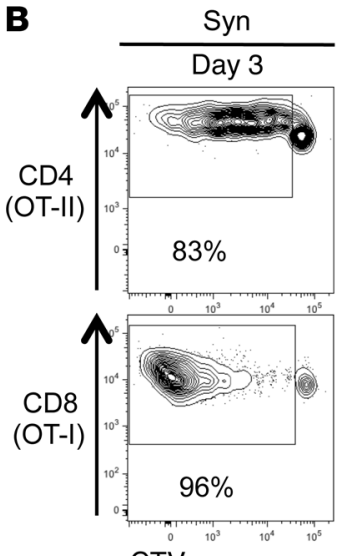

CTV
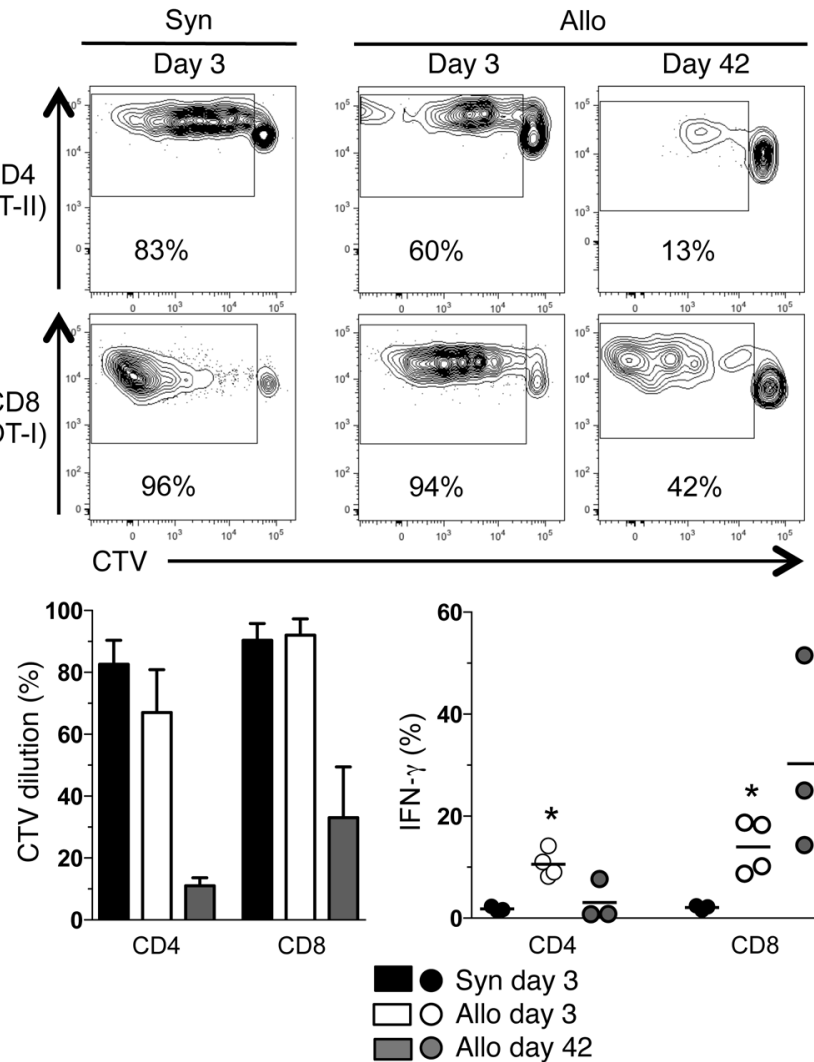

Figure 5. Graft mono-DCs present antigen to CD4 and CD8 T cells ex vivo. (A) T cell proliferation (dilution of CTV) and IFN- $\gamma$ production in response to contro splenic CD11 $b^{+}$DCs sorted from naive mice and fed LPS-free OVA in the presence or absence of exogenous LPS. (B) T cell proliferation and IFN- $\gamma$ production in response to graft mono-DCs fed LPS-free OVA in the absence of exogenous LPS. Mono-DCs were sorted from syngeneic heart grafts on day 3 after transplantation and from heart allografts on either day 3 or day 42. Same donor-recipient strain combination as in Figure 2, A-C. Flow cytometry was performed 5 days after in vitro stimulation of T cells with DCs. DC/T cell ratio $=1: 50$. Percentages indicate CFSE-diluted cells. ${ }^{*} P<0.05$ compared with the syngeneic group.

ure 4B). By day 3 , eGFP ${ }^{+}$cells had accumulated only in allogeneic grafts (Figure 4A), and most had exited the blood vessels (Figure 4B and Supplemental Video 1). In addition, they acquired dendritic morphology (Figure 4C). These results are consistent with the heart and bone marrow plug allograft data shown in Figures 2 and 3 and provide additional proof that monocyte migration and differentiation into DCs is enhanced in allogeneic tissues.

Mono-DCs from heart allografts present antigen and activate $T$ cells ex vivo. Innate sensing of microbes induces DCs to mature into potent APCs that drive $\mathrm{T}$ lymphocyte proliferation and differentiation. To test whether mature mono-DCs generated in response to allotransplantation acquire the same cardinal properties, we sorted mono-DCs from allogeneic heart grafts either 3 or 42 days after transplantation into $R A G^{-/-} \gamma^{-/-}$mice, and their capacity to process and present LPS-free OVA to OVA-specific CD4 (OT-II) and CD8 (OT-I) T cells was tested in vitro by measuring $\mathrm{T}$ cell proliferation and IFN- $\gamma$ production. As shown in Figure 5A, control splenic $\mathrm{CD} 11 \mathrm{~b}^{+} \mathrm{DCs}$ isolated from naive (untransplanted) mice did not present LPS-free OVA to either OT-I or OT-II cells unless adjuvant (LPS) was added to the culture. In contrast, mono-DCs sorted from day 3 allografts presented LPS-free OVA efficiently, causing both OT-I and OT-II cell proliferation and IFN- $\gamma$ production in the absence of exogenous LPS (Figure 5B). Mono-DCs isolated from day-42 allografts were also capable of presenting antigen with- out exogenous LPS, albeit less efficiently than those isolated from day-3 allografts (Figure 5B). They were also significantly better at activating CD8 (OT-I) than CD4 (OT-II) T cells (Figure 5B). MonoDCs isolated from day-3 syngeneic grafts and fed LPS-free OVA stimulated OT-I and OT-II proliferation but, unlike mono-DCs from allogeneic grafts, did not induce IFN- $\gamma$ production (Figure 5B). Therefore, allogeneic non-self acts as an immune adjuvant that causes persistent maturation of monocytes into APCs, which resemble in their function those induced by non-self, microbial ligands such as LPS (Figure 5A and refs. 31, 32). In contrast, acute inflammation alone (transplantation of syngeneic grafts) elicits transient maturation of monocytes into DCs that can stimulate $\mathrm{T}$ cell proliferation in response to cognate antigen but that do not induce IFN- $\gamma$ production.

Innate allorecognition by monocytes promotes $T$ cell-mediated graft rejection. We next asked whether innate allorecognition generates a productive adaptive immune response in vivo - that is, does it promote $\mathrm{T}$ cell-mediated graft rejection? To answer this question, we transplanted B6-OVA $\left(\mathrm{H}-2^{\mathrm{b}}\right)$ or CB6F1-OVA $\left(\mathrm{H}-2^{\mathrm{b} / \mathrm{d}}\right)$ heart grafts into $B 6 R A G^{-/ \gamma} \gamma c^{-/-}\left(\mathrm{H}-2^{b}\right)$ recipients and transferred OVA-specific, RAG-deficient OT-II T cells 2 days after transplantation. B6-OVA grafts differ by a single antigen (OVA) but are otherwise syngeneic to the recipient, while CB6F1-OVA grafts express OVA and at the same time are allogeneic to the host. In 

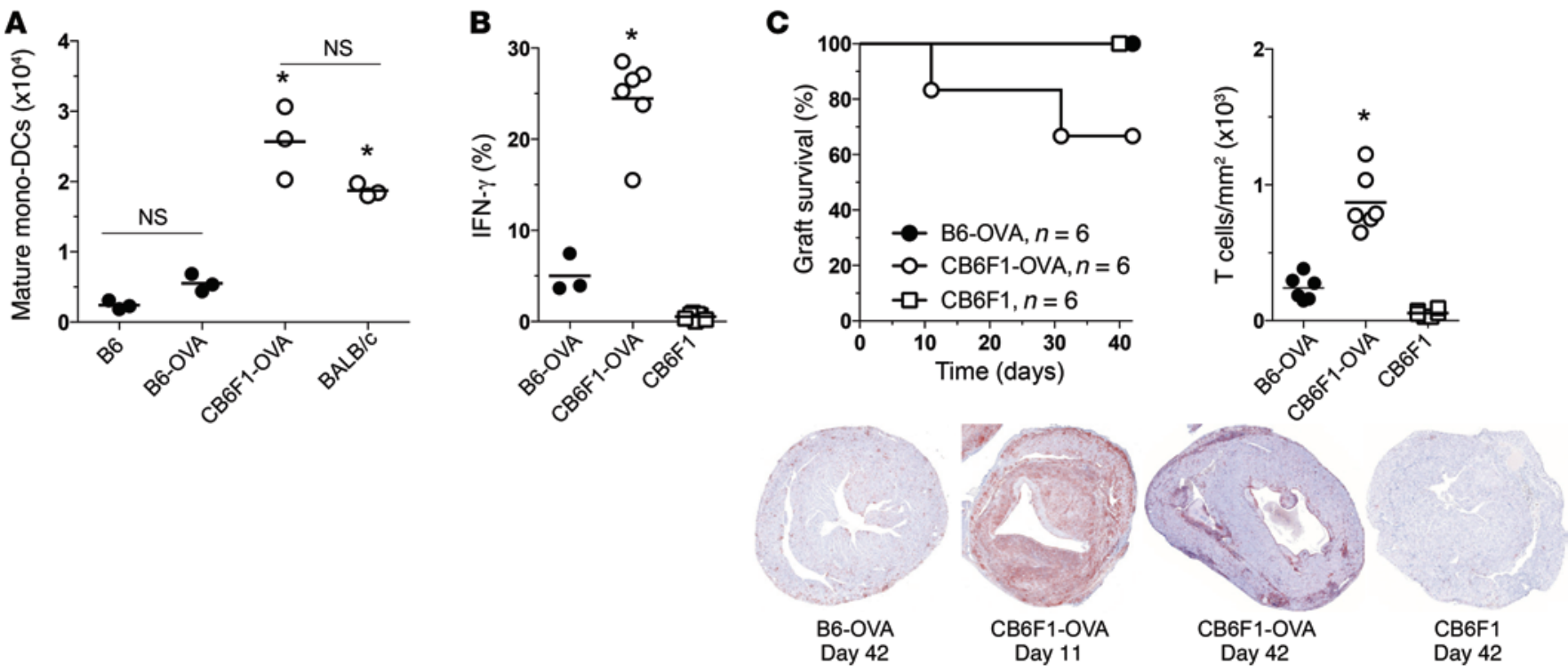

Figure 6. Innate sensing of allogeneic non-self precipitates graft rejection. (A) Heart grafts from multiple donor strains (shown on $x$ axis) were transplanted into $\mathrm{B} 6 \mathrm{RAG^{-1- }} \gamma \mathrm{c}^{-/-} \mathrm{C} X_{3} C R{ }^{19 f p /+}$ recipients, and host-derived mature mono-DCs present in the grafts were enumerated by flow cytometry 3 days later. (B and $\mathbf{C}$ ) Graft recipients, as in $\mathbf{A}$, received $5 \times 10^{5}$ OT-II cells 2 days after transplantation. Grafts and spleens were harvested on the day of rejection or at termination of the experiment (day 42). IFN- $\gamma$ production by OT-II cells in the spleen is shown in $\mathbf{B}$. Allograft survival, number of graftinfiltrating T cells, and representative graft sections stained with anti-CD3 (red) and hematoxylin (blue) are shown in C (original magnification, $\times 2$ ). ${ }^{*} P<0.05$ compared with B6 and B6-OVA groups.

both cases, antigen recognition by host $\mathrm{T}$ cells (consisting entirely of the transferred $R A G$-deficient OT-II cells) is restricted to a single OVA peptide that is presented in the context of host $\mathrm{MHC}\left(\mathrm{H}-2^{\mathrm{b}}\right)$, whereas host monocytes remain free to mount an innate response to allogeneic non-self (present on CB6F1-OVA but not B6-OVA grafts). As shown in Figure 6, B6-OVA grafts did not induce monocyte differentiation into DCs above that observed in syngeneic (B6) grafts (quantitated on day 3 in an independent group of mice that did not receive OT-II cells) (Figure 6A), caused minimal IFN- $\gamma$ production by OT-II cells in the spleen (Figure 6B), and were not rejected (Figure 6C). All grafts were still beating 42 days after OT-II cell transfer, and all exhibited minimal infiltration with $\mathrm{T}$ cells (Figure 6C) and no graft vasculopathy (chronic rejection). These findings imply that acute events associated with the transplantation procedure, such as danger signals induced by tissue ischemia, are not sufficient for generating a productive adaptive immune response that causes the rejection of a graft expressing a single non-self antigen (OVA). In contrast, CB6F1-OVA grafts, which are allogeneic to the host, induced significantly greater monocyte differentiation into DCs, comparable to that observed in BALB/c grafts (Figure $6 \mathrm{~A}$ ), stimulated IFN- $\gamma$ production by OT-II cells (Figure 6B), and underwent acute cellular rejection (2 of 6 grafts stopped beating on days 11 and 32 after OT-II cell transfer, and all exhibited substantial infiltration with T cells) (Figure 6C). Control CB6F1 grafts, which do not express OVA, did not elicit an OT-II IFN- $\gamma$ response (Figure 6B) and were not rejected (Figure 6C), confirming that acute rejection of $C B 6 F 1-O V A$ grafts did not result from cross-reactivity of OT-II T cells with $C B 6 F 1$ alloantigens. Therefore, as with microbial non-self, allogeneic non-self triggers an innate immune response that promotes $\mathrm{T}$ cell immunity.
Monocyte depletion blunts $T$ cell-mediated graft rejection in immunocompetent mice. To further investigate the in vivo relevance of allorecognition by monocytes, we tested the effect of depleting monocyte lineage cells on the antigraft $\mathrm{T}$ cell response in immunocompetent mice. B6 CD11bDTR chimeras (B6 mice reconstituted with syngeneic bone marrow cells that express the simian diphtheria toxin receptor [DTR] under the control of the $C D 11 b$ promoter) were transplanted with BALB/c hearts and administered diphtheria toxin (DT) to deplete bone marrowderived CD11 ${ }^{+}$cells. DT was restricted to 4 injections $(12.5 \mathrm{ng} / \mathrm{g}$ each) given on days $0,2,4$, and 6 after transplantation to avoid the morbidity and mortality associated with a high cumulative DT dose. Control chimeras (B6 mice reconstituted with syngeneic bone marrow cells that do not express DTR and transplanted with BALB/c hearts) received the same DT regimen. We found that DT reduced blood monocytes by approximately $70 \%$ in CD11b-DTR chimeras, while sparing lymphocytes and neutrophils (Supplemental Figure 3 and ref. 33). Grafts harvested on day 7 from these mice had significantly fewer $\mathrm{CD}^{+}, \mathrm{CD} 4^{+}, \mathrm{CD} 8^{+}$, and IF $-\gamma^{+}$T cells than did grafts from control chimeras (Figure 7, A and B). They also lacked mono-DCs and were less infiltrated with monocytes and neutrophils (Figure 7C). Reduction in the latter was likely a consequence of depleting monocytes, which are known to drive neutrophil entry into transplanted tissues (34). However, to test whether neutrophil depletion in the graft also contributed to blunting of the $\mathrm{T}$ cell response, we transplanted BALB/c heart grafts into wild-type B6 mice and treated the recipients with either $1 \mathrm{~A} 8$, an anti-mouse Ly6 G monoclonal antibody that specifically depletes neutrophils but spares monocytes (Supplemental Figure 3B and ref. 35), or isotype control antibody (2A3). As shown in Figure 7, D and E, grafts harvested 
A
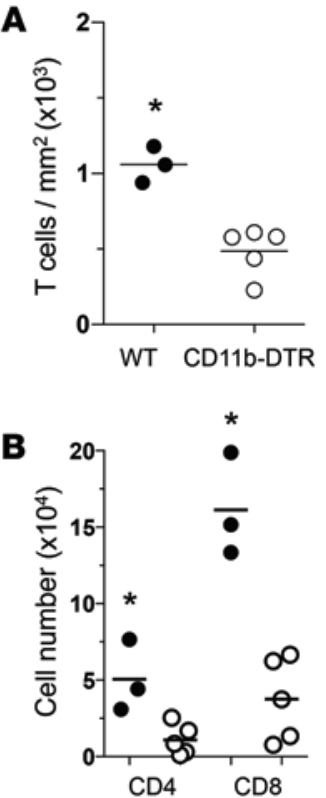
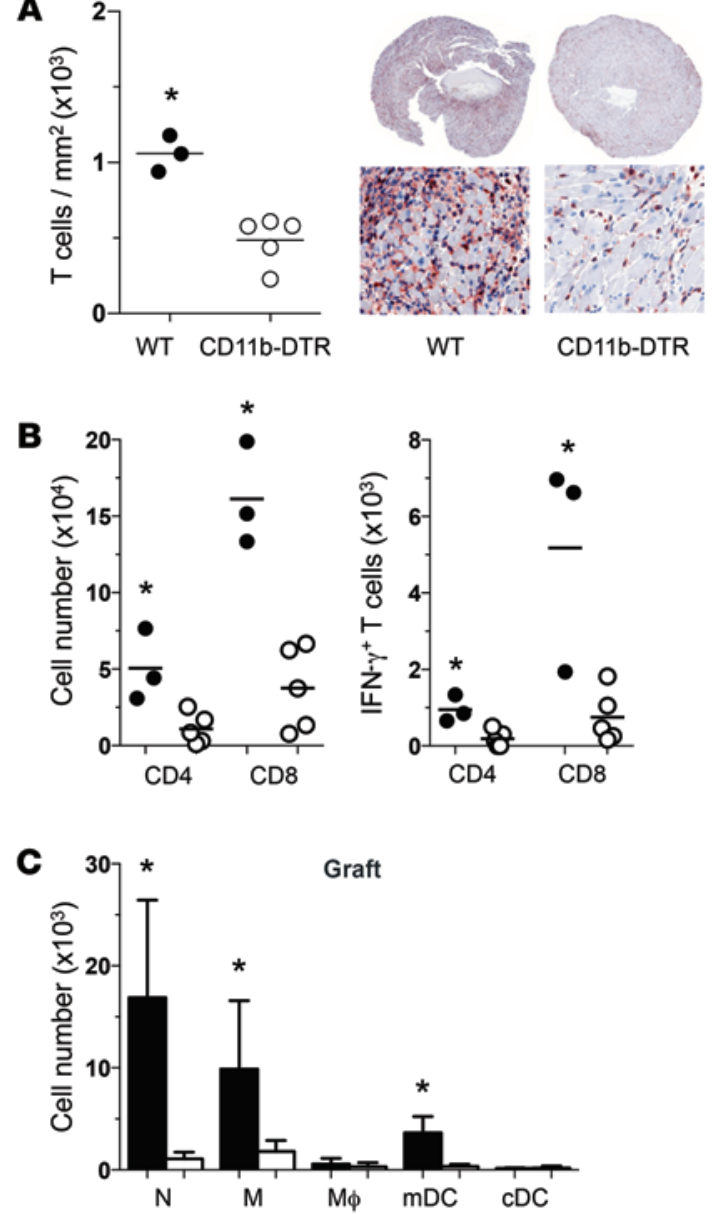
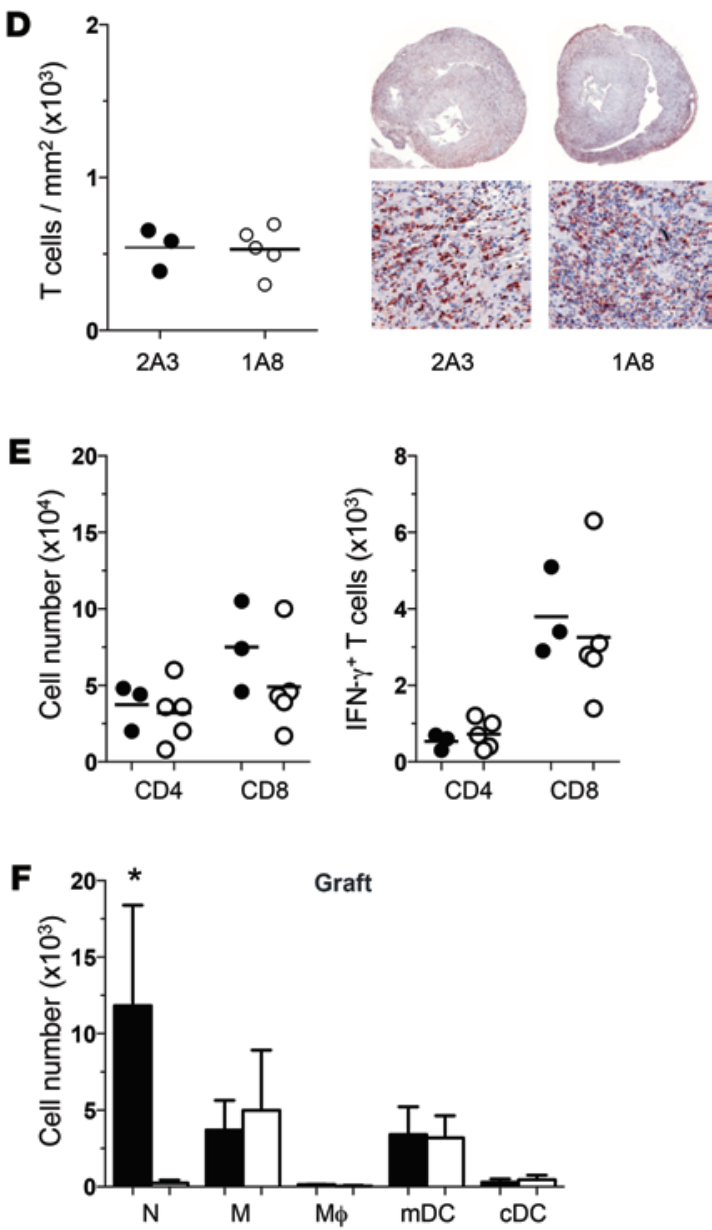

Figure 7. Monocyte depletion blunts T cell-mediated rejection. (A-C) B6 CD11b-DTR chimeras (white circles) and B6 wild-type (WT) control chimeras (black circles) were transplanted with BALB/c hearts and treated with $12.5 \mathrm{ng} / \mathrm{g}$ DT on days 0,2 , 4, and 6 . Grafts were harvested on day 7 after transplantation. (A) Enumeration of intragraft $\mathrm{CD3}^{+}$cells. Micrographs show graft sections stained with anti-CD3 (red). Original magnification, $\times 2$ (top panels) and $\times 40$ (bottom panels). (B) Flow cytometric enumeration of total intragraft CD4 ${ }^{+}$and CD8 ${ }^{+} \mathrm{T}$ cells (left panel) and IFN- $\gamma$-producing T cells (right panel). (C) Enumeration of neutrophils $(\mathrm{N})$, monocytes $(\mathrm{M})$, macrophages $(\mathrm{M} \phi)$, mono-DCs (mDC), and conventional DCs (cDC) in heart allografts. (D-F) Wild-type B6 mice were transplanted with BALB/c heart grafts and were treated with either $1 \mathrm{~A} 8$ (neutrophil-depleting anti-mouse Ly6C Ab; white circles) or 2A3 (isotype control antibody; black circles). The same enumeration was performed as in $\mathbf{A}-\mathbf{C}$. $n=3$ and 5 mice/group. ${ }^{*} P<0.05$ compared with control groups.

on day 7 from neutrophil-depleted mice had the same number of $\mathrm{CD}^{+}, \mathrm{CD}^{+}, \mathrm{CD}^{+}$, and IFN- $\gamma^{+}$T cells as did grafts from nondepleted (isotype control antibody-treated) recipients. In addition, neutrophil depletion did not reduce monocyte or monoDC infiltration of the grafts (Figure 7F). These findings provide strong evidence that mono-DCs promote $\mathrm{T}$ cell-mediated rejection in a physiological model of organ transplantation.

To further study the role of mono-DCs in the rejection process, we examined the effect of extended monocyte depletion on heart allograft survival and histology (Supplemental Figure 4). BALB/c hearts were transplanted into B6 CD11b-DTR or B6 wildtype bone marrow chimeras, and DT was administered at $25 \mathrm{ng} / \mathrm{g}$ i.p. every other day starting 1 day before transplantation. We observed significant DT-related morbidity in both groups because of the high cumulative dose administered. One wild-type recipient was sacrificed on day 6 because of morbidity, while the other 4 rejected their grafts by day 11 . All grafts exhibited dense T cell infiltration, consistent with acute cellular rejection. CD11b-DTR recipients were sacrificed between days 8 and 15, again because of morbidity, but all had strongly beating allografts at the time of sacrifice, and immunohistology demonstrated sparse T cell infiltration. These data suggest that monocyte depletion may delay graft rejection in otherwise unmanipulated mice.

MyD88 and NALP3 pathways are not necessary for host monocyte response to allografts. To test whether known innate signaling pathways that participate in danger recognition also participate in the monocyte response to allogeneic non-self, we transplanted BALB/c hearts into wild-type, $\mathrm{Myd} 88^{--}$and $\mathrm{Nalp}^{-/-} \mathrm{B} 6$ recipients and enumerated host mature mono-DCs in the grafts 3 days later. As shown in Figure 8, mono-DC infiltration of the allografts was significantly greater in all 3 recipient groups compared with that seen in the syngeneic (B6) grafts transplanted into wild-type $\mathrm{B} 6$ mice. No significant differences in mono-DC numbers were detected between allografts transplanted into knockout and wild-type hosts, although we observed a trend toward more infiltration in $\mathrm{Myd88^{-/ }}$ recipients. These results suggest that innate allorecognition of allogeneic nonself by monocytes involves signaling pathways distinct from those triggered by danger-associated molecules. 


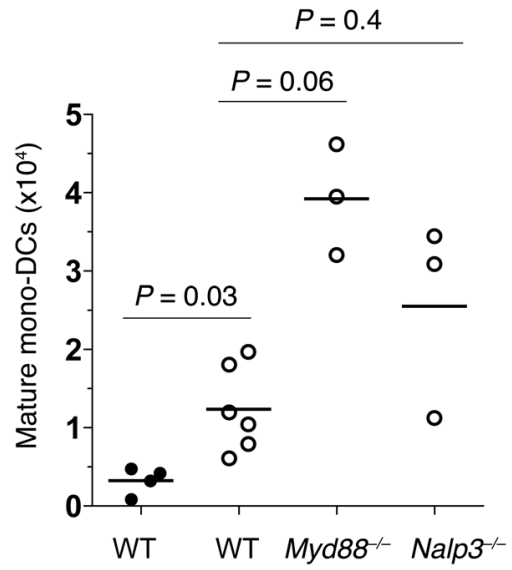

Figure 8. MyD88 and NALP3 pathways are not necessary for host monocyte response to allografts. Syngeneic heart grafts (B6; black circles) were transplanted into B6 wild-type mice. Allogeneic heart grafts (BALB/C; white circles) were transplanted into B6 wild-type, $\mathrm{Myd} 88^{-{ }^{--}}$, or $\mathrm{Nalp3}^{-1-}$ mice. Host mature mono-DCs that infiltrated the grafts were enumerated 3 days after transplantation. Recipient strains are indicated on the $x$ axis. $P$ values by Mann-Whitney $U$ test are shown.

\section{Discussion}

We have demonstrated that allogeneic tissues transplanted into lymphoid cell-deficient mice elicit innate responses that are quantitatively and qualitatively distinct from those elicited by syngeneic grafts. Allogeneic grafts induced persistent differentiation of monocytes into mature DCs that were present in greater numbers than in syngeneic grafts, expressed IL-12, and stimulated T cell proliferation and IFN- $\gamma$ production. In contrast, syngeneic grafts elicited transient differentiation of monocytes into mature DCs that neither expressed IL-12 nor stimulated IFN- $\gamma$ production. We also provided evidence that enhanced differentiation of monocytes into mature DCs in allografts potentiates $\mathrm{T}$ cell-mediated rejection. Therefore, allogeneic non-self links innate immunity to adaptive immunity by inducing the maturation of DCs.

Our findings unite alloimmunity with the Janeway model of microbial immunity, which states that recognition of non-self determinants underlies the initiation of protective immune responses (1). Traditionally, innate immune activation and APC maturation after tissue transplantation have been attributed to danger molecules released by dying graft cells, a known consequence of ischemia-reperfusion injury (11). If danger were the sole driver of innate immune activation, one would expect APC maturation to be identical in allogeneic and syngeneic grafts transplanted into lymphoid cell-deficient hosts, since both types of grafts are subjected to the same surgical procedures, and the contribution of the host's adaptive alloimmune response has been eliminated. However, we found that this is not the case. Monocyte-derived DC numbers, maturation, and function were significantly different between allogeneic and syngeneic grafts, and these differences had a demonstrable impact on $\mathrm{T}$ cell activation and graft rejection. Innate sensing of allogeneic non-self by monocytes, therefore, provides an important immune adjuvant effect above and beyond that provided by danger signals. Our data further indicate that the adjuvant effect of allogeneic non-self is necessary for inducing the IFN- $\gamma^{+}$Th1 immunity characteristic of most rejection responses.
The results reported in this article further establish the existence of allorecognition mechanisms within the innate immune system, a property previously attributed only to adaptive immune cells ( $\mathrm{T}, \mathrm{B}$, and NK cells). The possibility that innate immune cells respond to allogeneic non-self has been suggested before (36), but the relevance of such a response to protective immunity has not been elucidated. Harrison and colleagues identified a subtle difference in neutrophil and macrophage infiltration of the mouse peritoneal cavity after injection of allogeneic cells compared with injection of syngeneic cells (37). This difference was further exaggerated if xenogeneic cells were transplanted. We have previously reported that injection of allogeneic splenocytes into the ear pinnae of $R A G^{-/-}$mice elicits significantly greater swelling and infiltration of the skin with host myeloid cells than injection of syngeneic splenocytes (26). The response occurred independently of NK cells and was mediated by monocytes. These earlier studies set the stage for exploring innate allorecognition but fell short of defining how it affects the host. In contrast, we have established in the current study the physiological and disease relevance of innate allorecognition by demonstrating its role in triggering $\mathrm{T}$ cell-dependent alloimmunity and graft rejection. The determinants responsible for innate allorecognition, however, remain to be defined. Our observation that NOR and NOD grafts elicit markedly different monocyte responses despite sharing the same MHC suggests that polymorphic non-MHC loci that differ between NOR and NOD mice code for allodeterminants that modulate monocyte differentiation. Non-MHC regions that are not shared between the NOR and NOD strains contain many genes that code for immunologically relevant molecules. One such gene codes for SIRP $\alpha$, a polymorphic membrane protein expressed on monocytes, macrophages, DCs, and neurons (38). Engagement of SIRP $\alpha$ with its ligand CD47, which is ubiquitously expressed but is not polymorphic, delivers a "do-not-eat-me" signal to macrophages and inhibits DC maturation. CD47-SIRPa interactions have been shown to enhance engraftment of xenogeneic and allogeneic cells in mice and to protect tumor cells from phagocytosis (39-42). The availability of multiple NOD.NOR and NOR.NOD congenic mice should allow us in the future to determine through a classical genetic mapping strategy whether SIRP $\alpha$ or other gene(s) account for the observed innate response to allogeneic non-self.

The novel findings reported in this article provide the impetus to tackle vexing problems in organ transplantation, such as tolerance, through the fresh prism of innate allorecognition. Our key observation that monocyte recognition of allogeneic non-self persists over time (long after acute surgical inflammation has subsided) should also provide valuable insights into the pathogenesis and treatment of chronic rejection, the principal cause of longterm graft failure in humans, and into better strategies to achieve durable transplant tolerance.

\section{Methods}

Mice. C57BL/6J (B6) (Thy1.2, CD45.2, H-2 ${ }^{\mathrm{b}}$ ), BALB/cJ (BALB/c, H-2 ${ }^{\mathrm{d}}$ ), BALB.B (H-2 $\left.{ }^{\mathrm{b}}\right)$, B6.SJL-Ptprca Pepcb/BoyJ (CD45.1, Thy1.2, H-2 $)$, CByJ.

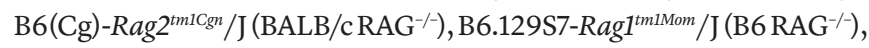
B6.129P-Cx3cr1 tmlitt $^{\text {J J }}$ (B6 CX CR1-eGFP CD45.2), B6.129P(Cg)-

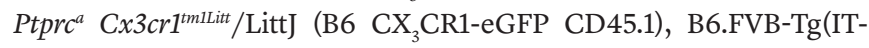
GAM-DTR/EGFP)34Lan/J (B6 CD11b-DTR), CB6F1/J (B6 x BALB/c F1, H-2 ${ }^{\mathrm{k} / \mathrm{d}}$ ), C57BL/6-Tg(CAG-OVA)916Jen/J (B6-OVA), NOD (NOD/ 
ShiLtJ), and NOD RAG ${ }^{-/} \gamma \mathrm{c}^{-/-}$(NOD.Cg-Rag1 ${ }^{\text {tmlMom }} \mathrm{Il} 2 \mathrm{rg}^{\text {tmlWjl }} / \mathrm{SzJ}$ ) mice were purchased from The Jackson Laboratory. B6.129S6-Rag2 ${ }^{\text {tmIFwa }}$ $\mathrm{Tg}$ (TcraTcrb) 425Cbn (RAG $\left.{ }^{-/} \mathrm{OT}-\mathrm{II}\right)$ and B10;B6-Rag2 ${ }^{\text {tmIFwa }}$ II2 $\mathrm{rg}^{\text {tmlwjl }}$ $\left(\mathrm{RAG}^{-/} \mathrm{\gamma c}^{-/-}\right.$) mice were purchased from Taconic. B6.129P-Cx3 $\mathrm{cr} 1^{\text {tmILitt }} / \mathrm{J}$ mice were bred on the $\mathrm{RAG}^{-/} \gamma^{-/-}$background. C57BL/6-Tg(TcraTcrb)1100Mjb/J (B6 OT-I; The Jackson Laboratory) were bred on the $\mathrm{RAG}^{-/-}$background. B6-OVA mice were crossed with BALB/c mice to generate $\mathrm{CB} 6 \mathrm{~F} 1-\mathrm{OVA}$ mice. $\mathrm{MyD} 88^{-/-}$and $\mathrm{NALP}^{-/-}$mice on the $\mathrm{B} 6$ background were bred and maintained at the University of Pittsburgh animal facility. All animals were maintained under specific pathogenfree conditions.

Surgical procedures. Heterotopic transplantation of primarily vascularized cardiac and kidney grafts was performed as previously described $(43,44)$. Cardiac allograft rejection was defined as cessation of a palpable heartbeat and was confirmed by histological analysis. Bone marrow plug transplantation was performed under the kidney capsule by isolating intact bone marrow plugs from donor femurs. Recipient mice were anesthetized and the kidney exposed via a small flank incision. A small incision was made in the kidney capsule and a pocket created with blunt forceps. The bone marrow plug was placed in the subscapular pocket with vascular forceps.

Bone marrow chimeras. Bone marrow chimeras were created by lethally irradiating B6 wild-type (CD45.1) mice (10 Gy total body irradiation using a Nordion Gamma Cell 40 cesium source) followed by i.v. transfer of $10^{7}$ bone marrow cells from either B6 wild-type (CD45.2) or B6 CD11b-DTR/eGFP (CD45.2) mice. Donor bone marrow reconstitution was verified 8 weeks after transplantation by flow cytometric analysis of peripheral blood.

Mouse treatment. Bone marrow chimeras were treated with 12.5 or $25 \mathrm{ng} / \mathrm{g}$ body weight DT (Sigma-Aldrich) at the indicated time points (Figure 7 and Supplemental Figure 4). For NK cell depletion, BALB/c $\mathrm{RAG}^{-/-}$mice (heart donors) received $50 \mu \mathrm{l}$ of polyclonal rabbit antimouse Asialo GM1 antibody (Cedarlane) on days -2 and -1 before transplantation according to the manufacturer's instructions. NK cell depletion was verified in the spleen at the time of heart procurement for transplantation. Absence of transferred NK cells was also confirmed in the recipient blood and spleen at the time of graft harvest. For neutrophil depletion, mice received $500 \mu \mathrm{g}$ anti-mouse Ly-6G monoclonal antibody (clone 1A8) (35) or $500 \mu \mathrm{g}$ isotype control antibody (clone 2A3; BioXCell) daily i.p. starting on day -2 before transplantation and for the duration of the experiment.

Leukocyte isolation. Mice were anesthetized and perfused with 20 to $40 \mathrm{ml} \mathrm{PBS}$ plus $0.5 \%$ heparin via the left ventricle until the fluid exiting the right ventricle did not contain any visible blood. Cells from cardiac grafts were isolated as previously described (45). Briefly, tissues were homogenized using a GentleMACS tissue processor (Miltenyi Biotec) and digested at $37^{\circ} \mathrm{C}$ for 45 minutes in RPMI plus $10 \% \mathrm{FCS}$ containing Collagenase IV (350 U/ml; Sigma-Aldrich) and DNAse I (20 ng/ml, Sigma-Aldrich). Leukocytes were isolated by gradient centrifugation using Lympholyte M (Cedarlane). Total recovered cells were counted using a hemocytometer or an automated cell counter (Beckman Coulter). Leukocytes from bone marrow plugs were isolated using the same procedure.

Flow cytometry and intracellular cytokine staining. The following fluorochrome- or biotin-tagged antibodies were purchased from BD Pharmingen, eBioscience, BioLegend, or R\&D Systems: CD4 (RM4-5), CD8a (53-6.7), CD62L (MEL-14), CD44 (1M7), CD90.1 (OX-7),
CD90.2 (30-H12), CD45.1 (A20), CD45.2 (104), CD45R/B220 (RA36B2), CD49b (DX5), F4/80 (BM8), CD11b (M1/70), CD11c (N418), Ly-6C (HK1.4), Ly-6G (1A8), CD19 (1D3), MHCII (M5/114.15.2), CD80 (16-10A1), IL-12p40 (C17.8), TNF- $\alpha$ (MP6-XT22), and IFN- $\gamma$ (XMG1.2). Fixable live/dead Aqua cell stain (405 nm excitation) and Cell Trace Violet (405 $\mathrm{nm}$ excitation) were purchased from Invitrogen. Intracellular cytokine staining was performed as previously described (45). Briefly, cells were washed, stained for surface markers, fixed with BD Cytofix/Cytoperm, permeabilized with $0.25 \%$ saponin, and incubated with anti-IFN- $\gamma$, anti-TNF- $\alpha$ or anti-IL-12p40 antibody for 1 hour at room temperature. Flow acquisition was performed on an LSR Fortessa flow cytometer (BD Biosciences), and data were analyzed using FlowJo software (Tree Star Inc.).

Ex vivo antigen presentation assay. Mono-DCs (lineage [CD19, CD90, NK1.1, DX5] ${ }^{-} \mathrm{Ly}-6 \mathrm{G}^{-} \mathrm{CD} 45^{+} \mathrm{CD} 11 \mathrm{~b}^{+} \mathrm{GFP}^{+} \mathrm{F} 4 / 80^{\text {lo }} \mathrm{CD} 11 \mathrm{c}^{+}$) were sorted by flow cytometry from syngeneic and allogeneic grafts (control splenic

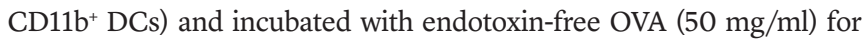
24 hours. For controls, CD11b ${ }^{+}$DCs were sorted from naive (untransplanted) mouse spleens and incubated with OVA alone or with OVA and LPS (100 ng/ml). CFSE or CellTrace Violet-labeled (CTV-labeled) OT-II (CD4) TCR-tg (Thy 1.2) and OT-I (CD8) TCR-tg (Thy 1.1) T cells were then added to the cultures $(50,000$ cells/well each; $1: 50 \mathrm{DC} / \mathrm{T}$ cell ratio) and harvested 4 days later. Brefeldin A was added 24 hours before harvesting. Cells were stained for CD4, CD8, congenic markers (Thy1.2 and Thy1.1), CD44, CD62L, and IFN- $\gamma$ and analyzed by flow cytometry.

2-photon intravital microscopy. Multiphoton intravital microscopy was performed on transplanted kidneys using an established method (44). An Olympus FluoView FV1000 microscope equipped with a Mai Tai DeepSee femtosecond-pulsed laser (Spectra-Physics) tuned and mode-locked to $915 \mathrm{~nm}$ was used for all experiments. Mice were anesthetized with isoflurane and oxygen and core body temperature maintained at $37^{\circ} \mathrm{C}$ with a homeothermic controller (TC-1000; CWE Inc.). Animals were kept hydrated by injecting $1 \mathrm{ml}$ of $5 \%$ dextrose lactated ringer's solution s.c. every 60 minutes. Blood vessels were visualized by injecting nontargeted QTracker 705 quantum dots (Life Technologies). The kidney graft was extraverted from the abdominal cavity with intact vascular connection and immobilized in a custom cup mount. A coverslip was placed on top of kidney, and $z$ stacks were visualized with a $\times 25$ water immersion objective (NA: 1.05) 25-50 $\mu \mathrm{m}$ below the kidney capsule. A total of 11 slices per stack were acquired at a step size of $2.5 \mu \mathrm{m}$. Brightness and laser power were adjusted based on the imaging depth and kept below phototoxic levels. Dwell time was set to $8 \mu \mathrm{s} /$ pixel and a resolution of $512 \times 512$ pixels. Approximately 30 -second-long stacks were repeatedly scanned up to 60 times for a maximum imaging time of 30 minutes per location. Up to 5 different locations per kidney graft were imaged. All acquired movies were analyzed using Imaris software (Bitplane). Drift was corrected using the blood vessels as a reference point. All $\mathrm{GFP}^{+}$cells detected during the imaging time were enumerated. Cells were determined to be extravascular if the majority or all of the cell body had moved outside the capillary lumen.

Immunohistochemistry. Grafts were harvested from mice after extensive cardiac perfusion and processed immediately by slicing into 2- to 3-mm sections and fixing in zinc-buffered formalin for 24 hours. Tissue was then paraffin embedded, and 5-mm sections were mounted on glass slides according to standard methods. Sections were stained with anti-mouse CD3 monoclonal antibodies and peroxidase-conjugated secondary antibody and scanned by a 
Zeiss digital microscopy system. $\mathrm{CD}^{+}$cells were enumerated using Pannoramic Viewer (3DHISTECH) and ImageJ (NIH) software.

Statistics. Four animals per group were chosen so that the experiment had $80 \%$ power to detect, at $5 \%$ significance, a 2 -fold difference, assuming a 0.5 -fold, between-variability difference. Mice from different litters were randomly assigned to the different experimental groups. Blinding was not possible. Donor-recipient pairs were sex matched. Both sexes of mice were used. Technical failures (nonbeating heart during the first 2 days after transplantation or nonperfused kidney graft at the time of intravital imaging) after transplantation were excluded from the analysis. All figures display the mean values with SD error bars. All calculations were performed using GraphPad Prism 6.0 (GraphPad Software). Statistical significance $(P<0.05)$ was calculated using an unpaired 2-sided $t$ test or the Mann-Whitney $U$ test.

Study approval. Animals were maintained and procedures were performed with approval of the Institutional Animal Care and Use Committee of the University of Pittsburgh.

\section{Acknowledgments}

We thank Jiyun Kim and the staff of the Intravital Imaging Facility at the Starzl Transplantation Institute of the University of Pittsburgh for valuable support and advice on 2-photon microscopy and Matthew Nicotra (University of Pittsburgh) for critical scientific advice. This work was supported by American Heart Association grant 11SDG7230011 (to M.H. Oberbarnscheidt); NIH grants (AI099465, to F.G. Lakkis, and AI064343, to F.G. Lakkis, W.D. Shlomchik, and D.M. Rothstein); and by the Frank and Athena Sarris Chair in Transplantation Biology (to F.G. Lakkis).

Address correspondence to: Fadi G. Lakkis, University of Pittsburgh, Thomas E. Starzl Transplantation Institute, Biomedical Science Tower W1548, 200 Lothrop Street, Pittsburgh, Pennsylvania 15261, USA. Phone: 412.383.5774; Fax: 412.383.9990; E-mail: lakkisf@upmc.edu.
1. Janeway CA Jr. Approaching the asymptote? Evolution and revolution in immunology. Cold Spring Harb Symp Quant Biol. 1989;54(pt 1):1-13.

2. Iwasaki A, Medzhitov R. Regulation of adaptive immunity by the innate immune system. Science. 2010;327(5963):291-295.

3. Klein J. Biology of the Mouse Histocompatibility-2 Complex. New York, Heidelberg, Berlin: Springer-Verlag; 1975:521-536.

4. Hildemann WH, Thoenes GH. Immunological responses of Pacific hagfish. I. Skin transplantation immunity. Transplantation. 1969;7(6):506-521.

5. Sykes M, Wood K, Sachs DH. In: Paul WE, ed. Fundamental Immunology. Philadelphia, Pennsylvania, USA: Woleters Kluwer/Lippincott Williams \& Wilkins; 2008:1426-1488.

6. Herzenberg L, Bianchi DW, Schroder J, Cann H, Iverson M. Fetal cells in the blood of pregnant women: detection and enrichment by fluorescence-activated cell sorting. Proc Natl Acad Sci US A. 1979;76(3):1453-1457.

7. Bonney EA, Matzinger P. The maternal immune system's interaction with circulating fetal cells. Jimmunol. 1997;158(1):40-47.

8. Pearse AM, Swift K. Allograft theory: transmission of devil facial-tumour disease. Nature. 2006;439(7076):549.

9. Murgia C, Pritchard J, Kim S, Fassati A, Weiss R Clonal origin and evolution of a transmissible cancer. Cell. 2006;126(3):477-487.

10. Gallucci S, Lolkema M, Matzinger P. Natural adjuvants: endogenous activators of dendritic cells. Nat Med.1999;5(11):1249-1255.

11. Kono H, Rock KL. How dying cells alert the immune system to danger. Nat Rev Immunol. 2008;8(4):279-289.

12. Iyer SS, et al. Necrotic cells trigger a sterile inflammatory response through the Nlrp3 inflammasome. Proc Natl Acad Sci U S A. 2009;106(48):20388-20393.

13. Shi Y, Evans JE, Rock KL. Molecular identification of a danger signal that alerts the immune system to dying cells. Nature. 2003;425(6957):516-521.

14. Shi Y, Galusha SA, Rock KL. Cutting edge: elimination of an endogenous adjuvant reduces the activation of CD8 $\mathrm{T}$ lymphocytes to transplanted cells and in an autoimmune diabetes model. JImmunol. 2006;176(7):3905-3908.

15. Sims GP, Rowe DC, Rietdijk ST, Herbst R, Coyle AJ. HMGB1 and RAGE in inflammation and cancer. Annu Rev Immunol. 2010;28:367-388.

16. Goldstein DR, Tesar BM, Akira S, Lakkis FG. Critical role of the Toll-like receptor signal adaptor protein MyD88 in acute allograft rejection. J Clin Invest. 2003;111(10):1571-1578.

17. Tesar BM, Zhang J, Li Q, Goldstein DR. TH1 immune responses to fully MHC mismatched allografts are diminished in the absence of MyD88, a toll-like receptor signal adaptor protein. Am J Transplant. 2004;4(9):1429-1439.

18. McKay D, Shigeoka A, Rubinstein M, Surh C, Sprent J. Simultaneous deletion of MyD88 and Trif delays major histocompatibility and minor antigen mismatch allograft rejection. Eur J Immunol. 2006;36(8):1994-2002.

19. Hutton MJ, Westwell-Roper C, Soukhatcheva G, Plesner A, Dutz JP, Verchere CB. Islet allograft rejection is independent of toll-like receptor signaling in mice. Transplantation. 2009;88(9):1075-1080.

20. Li H, et al. Graft-versus-host disease is independent of innate signaling pathways triggered by pathogens in host hematopoietic cells. JImmunol. 2011;186(1):230-241.

21. Bingaman AW, et al. Vigorous allograft rejection in the absence of danger. JImmunol. 2000;164(6):3065-3071.

22. Anderson C, Carroll J, Gallucci S, Ridge J, Cheever A, Matzinger P. Testing time-, ignorance-, and danger-based models of tolerance. J Immunol. 2001;166(6):3663-3671.

23. Anderson CC, Matzinger P. Immunity or tolerance: opposite outcomes of microchimerism from skin grafts. Nat Med. 2001;7(1):80-87.

24. Chan WF, Perez-Diez A, Razavy H, Anderson CC. The ability of natural tolerance to be applied to allogeneic tissue: determinants and limits. Biol Direct. 2007;2:10.

25. Zecher D, et al. NK cells delay allograft rejection in lymphopenic hosts by downregulating the homeostatic proliferation of $\mathrm{CD}^{+} \mathrm{T}$ cells.
JImmunol. 2010;184(12):6649-6657.

26. Zecher D, van Rooijen N, Rothstein D, Shlomchik W, Lakkis F. An innate response to allogeneic nonself mediated by monocytes. JImmunol. 2009;183(12):7810-7816.

27. Auffray C, Sieweke M, Geissmann F. Blood monocytes: development, heterogeneity, and relationship with dendritic cells. Annu Rev Immunol.2009;27:669-692.

28. Jung S, et al. Analysis of fractalkine receptor CX(3)CR1 function by targeted deletion and green fluorescent protein reporter gene insertion. Mol Cell Biol. 2000;20(11):4106-4114.

29. Auffray C, et al. Monitoring of blood vessels and tissues by a population of monocytes with patrolling behavior. Science. 2007;317(5838):666-670.

30. $\mathrm{Ko} \mathrm{S}$, et al. Bone marrow aplasia induced by passenger leukocytes from heart allografts. Exp Hematol. 2001;29(3):339-344.

31. León B, López-Bravo M, Ardavín C. Monocyte-derived dendritic cells formed at the infection site control the induction of protective Thelper 1 responses against Leishmania. Immunity. 2007;26(4):519-531.

32. Cheong $\mathrm{C}$, et al. Microbial stimulation fully differentiates monocytes to DC-SIGN/CD209(+) dendritic cells for immune T cell areas. Cell. 2010;143(3):416-429.

33. Duffield JS, et al. Selective depletion of macrophages reveals distinct, opposing roles during liver injury and repair. J Clin Invest. 2005;115(1):56-65.

34. Kreisel D, et al. In vivo two-photon imaging reveals monocyte-dependent neutrophil extravasation during pulmonary inflammation. Proc Natl Acad Sci U S A. 2011;107(42):18073-18078.

35. Daley JM, Thomay AA, Connolly MD, Reichner JS, Albina JE. Use of Ly6G-specific monoclonal antibody to deplete neutrophils in mice. J Leukoc Biol. 2008;83(1):64-70.

36. Fox-Marsh A, Harrison LC. Emerging evidence that molecules expressed by mammalian tissue grafts are recognized by the innate immune system. JLeukoc Biol. 2002;71(3):401-409.

37. Fox A, Mountford J, Braakhuis A, Harrison LC. Innate and adaptive immune responses to non- 
vascular xenografts: evidence that macrophages are direct effectors of xenograft rejection. Jimmunol. 2001;166(3):2133-2140.

38. Barclay AN, van den Berg TK. The interaction between signal regulatory protein alpha (SIRPa) and CD47: structure, function, and therapeutic target. Anпи Rev Immunol. 2014;32:25-50.

39. Takenaka K, et al. Polymorphism in Sirpa modulates engraftment of human hematopoietic stem cells. Nat Immunol. 2007;8(12):1313-1323.

40. Wang H, Wu X, Wang Y, Oldenborg PA, Yang
YG. CD47 is required for suppression of allograft rejection by donor-specific transfusion. J Immunol. 2010;184(7):3401-3407.

41. Majeti R, et al. CD47 is an adverse prognostic factor and therapeutic antibody target on human acute myeloid leukemia stem cells. Cell. 2009;138(2):286-299.

42. Tseng D, et al. Anti-CD47 antibody-mediated phagocytosis of cancer by macrophages primes an effective antitumor T-cell response. Proc Natl Acad Sci U S A. 2013;110(27):11103-11108.
43. Corry RJ, Winn HJ, Russel PS. Primarily vascularized allografts of hearts in mice: The role of $\mathrm{H}-2 \mathrm{D}, \mathrm{H}-2 \mathrm{~K}$, and non $\mathrm{H}-2$ antigens. Transplantation. 1973;16(4):343-350.

44. Camirand G, et al. Multiphoton intravital microscopy of the transplanted mouse kidney. Am J Transplant. 2011;11(10):2067-2074.

45. Oberbarnscheidt MH, et al. Memory T cells migrate to and reject vascularized cardiac allografts independent of the chemokine receptor CXCR3. Transplantation. 2011;91(8):827-832. 